\title{
Semblance or Similarity? Reflections on Simulation and Similarity
}

\author{
Jay Odenbaugh*
}

\section{Introduction}

Regarding the philosophical literature on models and modeling, Michael Weisberg writes,

What this new literature about modeling lacks, however, is a comprehensive account of the models that figure in the practice of modeling. (Weisberg, 2013, 4)

Simulation and Similarity provides such a comprehensive account of models. Specifically, the work provides an account of what models are (interpreted structures), the different types of model structures found in the sciences (concrete objects, mathematical objects, and computational structures), the types of modeling (target-directed, generalized, hypothetical, and targetless modeling), the relationships between different types of idealizations (Galilean, minimalist, and multiple models) and representational ideals (COMPleteness, Simplicity, 1-CAUSAl, MAXOUt, and P-GENERALity), the relationship between models and their targets (weighted feature-matching account of similarity), and an examination of robustness analysis (parameter, structural and representational robustness). The positions articulated are original, illuminating, and merit serious consideration and discussion by philosophers of science and scientists themselves. One of its admirable features is that are a few models used regularly throughout the text - the Lotka-Volterra predator-prey model, the San Francisco Bay model, and the

*Department of Philosophy, Lewis \& Clark College, 0615 SW Palatine Hill Rd, Portland OR, 97202, jay@lclark.edu 
Schelling model. This gives one a good sense of how models are constructed, analyzed, and evaluated by building familiarity with the same ones throughout. Additionally, it can be read by a wide variety individuals be they philosophers or scientists. But, obviously I came not to praise, so I turn to issues of disagreement and where more light should be cast.

Weisberg notes that he has offered a "model of modeling," he writes, "But just as in a representation of any other complex phenomenon, philosophical analysis will necessarily be partial and incomplete" (Weisberg, 2013, 6). As such, it will only be similar to the phenomena in certain degrees and in certain respects; it will abstract and idealize possibly distorting the target for various purposes. However, this implies that his account may be but a semblance of scientific practice. Below I explore just how much distortion is present.

\section{Ontology, Models, and Mathematics}

According to Weisberg, a model is an interpreted structure. He claims we should distinguish between three types of structures - mathematical, concrete, and computational. For example, the Lotka-Volterra predator-prey model is partly a mathematical object, the San Francisco Bay model is in part by a concrete object, and the Schelling model is in part by a computational structure. ${ }^{1}$ However, these models are only objects or structures in part. What structures lack which models possess is an interpretation (Weisberg, 2013, 15). An interpretation is what he terms a construal which includes an assignment, an intended scope, and fidelity criteria. The modeler stipulates what parts of the structure or object denote which parts of the target. Of course, not every property of the structure denotes some property of the target nor are all properties of the target denoted by some property of the

\footnotetext{
${ }^{1}$ Objects and structures are different if recent philosophy of mathematics is correct. Following Zermelo, we can define the natural numbers as $0=\emptyset, 1=\{\emptyset\}, 2=\{\{\emptyset\}\}$, and more generally $n=\{n-1\}$. Following von Neumann, we can define the natural numbers as $0=\emptyset, 1=\{\emptyset\}, 2=\{\emptyset,\{\emptyset\}\}$, and more generally $n=\{0,1, \ldots, n-1\}$. It follows that on first proposal that $2 \notin 4$ and on the latter $2 \in 4$ (Benacerraf, 1965). Mathematical structuralists note that both proposals satisfy Peano's axioms and claim the natural numbers, and all mathematical structures, are not objects - which objects? - but "positions" in a pattern (Resnik, 1997; Shapiro, 1997; Hellman, 1989). Following Weisberg, I will speak indiscriminately between objects and structures even if they are importantly different.
} 
structure. Additionally, they implicitly or explicitly specify fidelity criteria for the model to adequately represent target system's "surface" properties (dynamic fidelity) or "deep" causal structure (representational fidelity).

Consider one of Weisberg's chosen models, the Lotka-Volterra predatorprey model. Suppose that $V$ is prey abundance, $P$ is predator abundance, $r$ is the rate of increase of the prey independent of the predator, $a$ is the capture rate of prey by predator, $b$ is the conversion efficiency rate of prey captured by predators into new predators, and $q$ is the mortality rate of the predator independent of the prey. If make further assumptions regarding the functional form of the equations, we can then represent our predator-prey as follows,

$$
\begin{aligned}
& \frac{d V}{d t}=r V-a V P \\
& \frac{d P}{d t}=b a V P-q P
\end{aligned}
$$

On his view, we have provided an assignment to a mathematical object which can be similar to a target system or type of target system. Note that (1) and (2) do not describe a token or type of predator-prey system. They describe the model. The model, the interpreted mathematical structure, if it is an adequate representation, resembles the target system in accordance with the fidelity criteria. Weisberg is very clear on this point. He writes,

When we talk about models, write about them, or show a picture or diagram, we are employing a model description. These descriptions must be distinguished from the models themselves. (Weisberg, 2013, 33)

As another example,

Equations or other kinds of statements specify mathematical objects and these objects satisfy their descriptions. However, unlike in the case of concrete models, mathematical models can be studied and manipulated only via their descriptions. While the Lotka-Volterra model itself is not a set of equations, it can be studied only through proxies such as these equations. This is probably the main reason that scientists often informally refer to equations as models; their attention is focused on these equations. (Weisberg, 2013, 37) 
The first question I have concerns whether the distinction between types of structures mentioned above is really substantive. Relational structures consist in a set of objects with relations on them. So, if models are relational structures, then a model $M$ would be an ordered pair of objects $D$ and relations $R ; M=<D, R>$. In the three cases above, we have relational structures which differ with regard to the objects or relations included. A mathematical model takes a mathematical object like the real numbers and defines a set of mathematical relations, e.g. functions, on them. These functions are what are captured by equations like (1) and (2). A concrete model is a set of concrete objects with "physical" relations on them which include the intrinsic and extrinsic properties of interest. Finally, a computational model is a set of states and a set of procedures/algorithms, e.g. transition rules, defined on them. The is a straightforward unity amongst models. They are interpreted relational structures.

Weisberg never examines whether models are interpreted relational structures. This view does not imply concrete models are of interest only because of their mathematical properties nor denies that we "compare models to targets in structural, behavioral, and other nonobviously mathematical ways" (Weisberg, 2013, 21). It would still be true that "concrete models are much richer in properties than just the mathematical ones" (Weisberg, 2013, 21) since there is no restriction on what $R$ includes if $D$ is a set of concrete particulars. $^{2}$ He might think that the above proposal conflates how mathematical and computational models explain.

However, I think it is important to distinguish between these mathematical models and computational models because of how they are used in giving scientific explanations. When one invokes a computational model to explain some phenomenon, one is typically using transition rules or algorithm as the explanans... Conversely, in the types of models I am calling mathematical models, the mathematical structure, or relationship among variables, carries the explanatory power. (Weisberg, 2013, 20)

But, there is no such difference. In a computational model, transition rules describe the relations between states. Likewise, in a mathematical model,

\footnotetext{
${ }^{2}$ It is not clear what is means for a concrete particular to have a mathematical property. Suppose Jill is first in line and Jack is second. Does Jill have the mathematical property being first in line? Or, is this simply a spatial relationship between Jill and Jack represented using mathematical concepts Field (1980)?
} 
equations describe the relations between variables. An explanans can be constructed from the states and variables or from the rules and equations. Computational and mathematical models are in the same boat. All of this is a friendly amendment to Weisberg's approach since it is says models really are interpreted relational structures.

Nevertheless, with or without my friendly amendment, there is a serious worry lurking behind Weisberg's proposal. Consider the following passage.

For my purposes, I need only to assume that it makes sense to talk about mathematical objects such as functions and state spaces and that these objects are identical to the equations we use to describe them. Any metaphysical theory, even a highly deflationary one, that allows us to make sense of these aspects of mathematical practice is sufficient for my purposes. Which of these theories is true, of course, is a much more complicated issue. (Weisberg, 2013, 29)

First, given that Weisberg claims models and model descriptions are distinct, he cannot accept a deflationary view where models are just model descriptions (e.g. equations). Second, if models are distinct from model descriptions and there are mathematical models, then Weisberg is committed to the existence of mathematical objects and hence mathematical realism. Now, one might think "So what?"; why bind one's position on models and modeling to a compatibility with mathematical anti-realism? The third worry is that Weisberg's mathematical realism is incompatible with his account of the model-world relation.

Consider a simple model of a pendulum,

$$
m\left(\frac{d^{2} x}{d t^{2}}\right)=-\left(\frac{m g}{l}\right) x
$$

where $x$ is position, $m$ is mass, $g$ is the gravitational constant, and $l$ is the length of the pendulum. For a pendulum with very little friction, with small angles of swing, and very short time intervals, there are fidelity criteria such that this model can adequately represent a pendulum's oscillations. However, on Weisberg's view, this requires that the interpreted mathematical object and pendulum are relevantly similar and this is subject to what I will call Hughes' Objection. R.I.G. Hughes wrote, 
...[W]e may model an actual pendulum, a weight hanging by a cord, as an ideal pendulum. We may even be tempted to say that in both cases the relation between the pendulum's length and periodic time is approximately the same, and that they are in that respect similar to each other. But the ideal pendulum has no length, and there is no time in which it completes an oscillation. It is an abstract object, similar to material pendulums in no obvious sense (Hughes, 1997, 330) ${ }^{3}$

An object can have the properties periodic time and length only if it is spatiotemporal. Mathematical objects are not spatiotemporal. Hence, they cannot have the properties periodic time and length. Two objects are similar only if they share properties. Mathematical objects and pendulums cannot share the properties periodic time and length. Therefore, they cannot be similar with respect to periodic time and length.

It is important to note two things. First, this argument generalizes to any target which is a concrete particular and model which is in part a mathematical object where the properties are had only by concrete particulars. Second, note the the account of similarity as sharing of properties is presupposed by Weisberg's own weighted feature matching account of similarity. Expressions in his account include $M_{a} \cap T_{a}$ and $M_{m} \cap T_{m}$; the intersection of model attributes $M_{a}$ and target attributes $T_{a}$ and the intersection of mechanisms between $M_{t}$ and $T_{t}$. If no attributes or mechanisms are shared, then his measure $S(m, t)=0$ since the numerator $\left|M_{a} \cap T_{a}\right|+\left|M_{m} \cap T_{m}\right|=0$ and the relevant terms in the denominator $\left|M_{a}-T_{a}\right|+\left|T_{a}-T_{m}\right|+\left|T_{m}-T_{a}\right| \neq 0$. That is, there attributes and mechanisms the model has the target lacks and vice versa. Thus, the claim, "This actual pendulum is similar in its period and length to a model described by (3)" is false and necessarily so. ${ }^{4}$

\footnotetext{
${ }^{3}$ Hughes to my knowledge is the first philosopher of science to press this objection. However, Martin Thomson-Jones (2010) has offered it as well with Toon (2012) following suit. The first philosopher, of course, was Plato. In Book VII of The Republic, "[The] science [of geometry] is in direct contradiction with the language employed by its adepts.... [They talk] of squaring and applying and adding and the like....whereas in fact the real object of the entire subject is...knowledge...of what eternally exists, not of anything that comes to be this or that at some time and ceases to be." (Cooper and Hutchinson, 1997)

${ }^{4}$ Mathematical objects, if they exist, can have non-essential properties. For example, suppose my favorite number is 76 . If the number 76 has the property of being Jay's favorite, it has it accidentally not essentially. Some might object that this is a "Cambridge property." Fair enough.
} 
Weisberg could respond to this argument in three ways it seems to me. First, he could claim that mathematical objects and structures are not abstract but concrete (Field, 1980). If mathematical entities are concrete, they could possess properties like periodic time and length. However, this is unlikely to work. Consider the claim that the set of real numbers is larger than the set of natural numbers even though both sets have an infinite number of members. If concrete particulars are its truthmakers, then must be lots and lots and lots of truthmakers (i.e. their cardinality must be least $2^{\aleph_{0}}$ !). As philosopher of mathematics Stewart Shapiro writes,

...[I]t seems reasonable to insist that there is some limit to the size of the physical universe. If so, then any branch of mathematics that requires an ontology larger than that of the physical universe must leave the realm of physical objects if these branches are not to be doomed to vacuity. Even with arithmetic, it is counterintuitive for an account of mathematics to be held hostage to the size of the physical universe (Shapiro, 1997, 86). ${ }^{5}$

Second, Weisberg could respond to Hughes' Objection by denying that mathematical objects exist. This fares no better than the first response. Necessarily, if $x$ and $y$ are similar, then $x$ and $y$ exist. Hence, if it is true that mathematical objects do not exist, then it is false to claim that mathematical objects are similar to empirical systems. Thus, Weisberg faces the following dilemma. With respect to properties like periodic time and length, either there are models which are mathematical objects in which case they cannot be similar to concreta or there are no models which are mathematical objects in which case they cannot be similar to concreta.

A third response is this. Hughes' Objection depends on the claim that mathematical objects cannot have the spatiotemporal properties that pendulums do. However, suppose that the target is represented as a relational structure. For example, if a our model $M=<D, R_{i}>$ and the target model is $M^{*}=<D^{*}, R_{i}^{*}>$, then they can share properties. For example, $M$ and $M^{*}$ can be isomorphic, homomorphic, etc. This it seems to me is Weisberg's best option, and in a different context, he seems to endorse it.

\footnotetext{
${ }^{5}$ In Hartry Field's nominalization of Newtonian mechanics, he claims we can replace mathematical objects with space-time points and regions. Whether space-time points and regions are concrete is another thing since they lack extension, mass, and "locations" (Resnik, 1997)
} 
But how can mathematical and computational models be compared to concrete targets? What possible similarities do they have? I think this objection can easily be resolved. Mathematical and computational models, as well as concrete models in some cases, are compared to mathematical representations of targets, not the targets themselves. Each state of the target is mapped to some mathematical space. In simple, dynamical models, the mapping is such that the major determinable properties (e.g., species abundance, pressure, time, temperature, etc.) of the target are mapped to dimensions of a state space, and specific states are mapped to points in this space. Now one interpreted mathematical object can be compared to another, and we avoid problems about comparing mathematical properties to concrete properties. (Weisberg, 2013, 95)

Still, mathematical and concrete objects still don't share properties. Consider an isomorphism between $M$ and $M^{*}$. There is a function $f$ such that $\left\langle o_{1}, \ldots, o_{n}\right\rangle \in R$ if and only if $\left.f\left(o_{1}\right), \ldots, f\left(o_{n}\right)\right\rangle \in R^{*}$. At best, we have shown that there is a second-order property of isomorphism shared between two relational structures. However, there is no $R$ or $R^{*}$ respectively such that an element of $D$ and $D^{*}$ both have it. Therefore, even if here are mappings between interpreted relational structures there are no shared spatiotemporal properties between mathematical and concrete objects. Additionally, Weisberg is no fan of isomorphism and partial isomorphism accounts of the modelworld relationship. Hence, his account of the model world relationship cannot rest on such shared second-order properties. ${ }^{6}$

From here, the path only darkens. One could claim that, at least in the mathematical case, "Models are created by their descriptions" (Weisberg, 2013, 33). How could writing down an equation create a mathematical object? Likewise, one could claim that we simply stipulate that elements of mathematical structures denote features of targets but this seems wholly

\footnotetext{
${ }^{6}$ Some of his criticisms of isomorphism apply mutatis mutandis to his account. If isomorphism cannot capture relationships between features of models and targets because it is relation between structures, then a set-theoretic similarity measure has the same problem since it is between sets (Weisberg, 2013, 139-40). Additionally, he worries that there are two many mappings from models to targets (i.e. target models). But, if modeler's intentions can pick out the contexually relevant similarities in the feature set, then they can pick out the contextually relevant mappings.
} 
mysterious. Besides presupposing mathematical realism, how can we stipulate that a non-spatial, non-temporal object stands for a concrete one? We can stipulate that an expression means such-and-such and that an equation represents some target but this is a very different matter.

In summary, Weisberg has committed himself to a form of mathematical realism. Second, the form of mathematical realism he has committed himself to makes its well nigh impossible for mathematical models to be similar to target systems. Third, the most plausible way out of the problem simply pushes it back by claiming that models are similar to target models but we are left with the same problem with how target models represent targets. ${ }^{7}$

\section{Fictionalism}

After the ruckus over mathematical objects, one might think that fictionalism about models looks very appealing. Weisberg is no fictionalist however. Though some of his criticisms of specific fictionalist positions have force, I am unconvinced that fictionalism about models in general has been shown false. There are variety of fictionalist views so let's consider a few of them.

The first view is the simple fictions account which says that, "mathematical models are imaginary systems that would be concrete if they were real" (Weisberg, 2013, 49). As purported confirmation of it, consider what John Maynard Smith writes (Maynard Smith, 1989, 22),

Imagine a population of replicating RNA molecules. There is some unique sequence, $S$, that produces copies at a rate $R$ : all other sequences produce copies at a lower rate, $r .$. A sequence produces an exact copy of itself with probability $Q$. If $x_{0}$ and $x_{1}$ are the numbers of copies of $S$ and non- $S$ respectively, then ignoring deaths, $d x_{0} / d t=R Q x_{0}, d x_{1} / d t=r x_{1}+R(1-Q) x_{0}$.

\footnotetext{
${ }^{7}$ As a radical revision, it would be interesting to see Weiberg's account coupled with a structuralist account of mathematics. Mathematical model descriptions would be statements which claim targets instantiate mathematical structures. There would be worries e.g. an idealized model could not be instantiated by a target system. Interestingly, Resnik and Shapiro are both ante re realists accepting that mathematical structures exist independent of instantiation. However, they think we can recognize simple patterns amongst concreta thus have a posteriori knowledge of them. Hellman's version is nominalistic - it is a "mathematical structuralism without structures." I am indebted to Elaine Landry for helping me think through modeling and mathematical structuralism.
} 
However, since Maynard Smith's imaginary population of RNA does not exist then it cannot be similar to any target system of interest. If they are to share properties, then they both must exist. Hence, they cannot be similar.

Weisberg considers three more detailed accounts of fiction, that of Lewis (1978), Walton (1990), and Levy (2012). On Lewis' account, $p$ is true in fiction $F$ if, and only if, the counterfactual " $p$ would have been true had $F$ been told as known fact" is true in every belief world of the author's community. A belief world of some community is any possible world where all the overt beliefs of the community are true. So, consider a world in which the stories of Sherlock Holmes are told as known fact and where the beliefs of Sir Arthur Conan Doyle and his compatriots are true. This is a world in which it is true that "Sherlock Holmes lives on Baker Street." Hence, it is fictionally true for us. Analogously, Maynard Smith's RNA model description is fictionally true insofar as they are asserted as known fact and the beliefs of Maynard Smith and his compatriots are true in at least one possible world. Now, like many philosophers, Lewis' modal realism leaves me with an "incredulous stare." I simply find it too epistemically costly to accept the existence of non-actual possible worlds of which we can have no epistemic access.

Kendall Walton's account of fiction in Walton (1990) is importantly different from Lewis' and involves props, principles of generation, and makebelieve. In a game of make-believe, there are props about which participants agree to certain conventions; i.e. principles of generation. When the props are present they make-believe certain states of affairs to be the case. For example, if we agree that tree stumps are bears or images of green slime on a movie screen are a monster, then when we see those props we make-believe that there are bears or monsters present respectively. In fact, we may even have quasi-emotions towards them such as quasi-fear towards the green slime. Moreover, in our respective games, it will be fictionally true that there are bears or monsters present respectively. Thus, props and principles of generation generate fictional truths. With respect to Maynard Smith's RNA model, presumably the prop is the model description and given the conventions amongst population geneticists we make-believe that they are true and they are in the relevant game of make-believe.

Weisberg's major objection to Roman Frigg's Waltonian fictionalism (Frigg, 2010) is this,

If mathematical models are games of make-believe, they dont re- 
semble anything in the physical world because they are scientists' mental states. Thus, Frigg has to give us an account of how we can learn about real targets from games of make believe. This is a nontrivial matter because now we are owed an account of how something inside a modelers head can be compared with the properties of a target. (Weisberg, 2013, 54)

There is a problem here but I think Weisberg has misdescribed it. His worry is how can a mental representation be similar to a target system. However, Waltonian fictionalism is not committed to that claim. Rather, the Waltonian fictionalist would claim that Maynard Smith is taking a certain attitude to a prop; specifically, his make-believing that the differential equations are true of RNA populations. If this is so, it is fictionally true that they are true of some RNA population. In effect, the model doesn't apply outside of the game to some target, rather, the target enters the game. Whatever we learn about it, we learn about it in the make-believe. ${ }^{8}$ The more serious objection is sometimes models like Maynard Smith's are explanatory and predictively accurate. However, if modeling is a form of make-believe, then this scientific success is make-believe as well. The predictive and explanatory success due to modeling only occurs in a game. This view invokes a "incredulous stare" too. ${ }^{9}$

The last fictionalist view Weisberg considers is Arnon Levy's de re account (Levy, 2012). We make-believe of some actual target that it has properties that it doesn't have. So, of some population of RNA, Maynard Smith make-believes that the model description truly describes it. One problem for this view is that there must be an object which we make-believe has some property. However, if there is no such object then we cannot makebelieve of it that it has certain properties. Otherwise, it is not a de re view. The question is then are there objects which modelers posit which are nonexistent? As one example, sometimes philosophers suggest that population

\footnotetext{
${ }^{8}$ This is reminiscent of Bas van Fraassen's claim that scientists be "theoretically immersed" in the scientific world picture (van Fraassen, 1980).

${ }^{9}$ Toon (2012) has recently defended a form of Waltonian fictionalism. He thinks we compare fictions and the world. For example, we might compare the periodic time of an make-believe pendulum and actual pendulum. We can do this, but why? Why think the fiction tell us anything about actual pendulums unless it actually the model descriptions "mimic" the world? The comparison even if possible is unmotivated. As with art, why compare fiction and the world unless the former says something true about the latter? And if this is so, the claims are true of both.
} 
geneticists posit infinite populations and then claim there are no such things. In response, the de re fictionalist could claim there are populations and we might make-believe that they are infinite in size. ${ }^{10}$ Thus, it seems that the de re view is more credible than either Lewis' or Walton's view applied to modeling.

Independent of these particular proposals, Weisberg provides four arguments against fictionalism about models - the argument from interscientist variation, the the limited representational capacity of fictions, the inability of fictions view to account for modeling practice, and variation in the face of modeling practice. Suppose we consider the Maynard Smith's equations as a prop for make-believe. Weisberg writes,

As proponents of the fictions account like to emphasize, mathematical descriptions are extremely sparse. If the mathematical description exhausted the focal properties of the model, then models would be correspondingly sparse in their portrayal of fictional scenarios. Fictions then cease to look at all like real-world scenarios, militating against the claim that models can be compared to real systems in a straightforward way. (Weisberg, 2013, 58)

However, as he notes, every fictionalist assumes there something like Lewis' belief world or Walton's principles of generation. Hence, there is more in a make-believe than just the prop of equations. Thus, there is no more reason to think that there is more problematic variation here than there is amongst viewers watching movies or reading novels. Sure, there will be differences of interpretation but this is true on Weisberg's view too. Consider how much debate there is over interpreting parameters in mathematical models. So, there is variation in both the sciences and the arts, but neither seems particularly objectionable. ${ }^{11}$

\footnotetext{
${ }^{10}$ I doubt we even need to be fictionalists regarding "infinite population size." Population geneticist are claiming that as $N$ gets larger and larger, the difference between actual and expected reproductive success becomes as small as you like. I owe this point to Michael Strevens.

${ }^{11}$ As a problem case, Weisberg thinks that one make-believes regarding a predator-prey system that it must include make-believing that it has a spatial arrangement (Weisberg, 2013, 60). But, this is not given by make-believing the model description is true. But insofar as one make-believe (1) and (2) are true this includes make-believing that the system has spatial arrangement given by the term $a V P$. They are make-believing the
} 
A second objection is that is the fictionalist cannot account for the different representational capacities of models. He writes,

Fictionalists regard the Lotka-Volterra model as an imaginary system composed of a predator population and a prey population. Setting aside, for a moment, how specific this has to be (are the predators sharks?), the model is composed of concrete, discrete organisms that interact with one another. But the equations used to describe the Lotka-Volterra model do so in terms of populations. This means that no individual organism is represented, in the mathematics, only populations of organisms. (Weisberg, 2013, 61)

According to Weisberg, the fictionalist considers "a model of predation has to be composed of concrete populations of discrete and distinct individuals" (Weisberg, 2013, 62). But, their fictional model must be of individual organisms. Individuals are precisely what the Lotka-Volterra model leaves out. This however misconstrues fictionalism. The fictionalist makes-believes that the model description is true. Thus, the model is silent regarding anything not specified by (1) and (2) and the common beliefs of the relevant community. They need not have any particular beliefs or make-beliefs about the "discrete and distinct individuals."

A third objection is that Fictionalism distorts modeling practice. According to Weisberg, modeling often involves indirect representation. One writes down a set of equations specifying a model and the model is relevantly similar to the target. However, the Fictionalist makes-believe of some object or objects that it has certain properties or some prop is true of it. Thus, it is a form of direct representation. First, making-believing create an opaque context and thus one can make-believe that $p$ without make-believing that $q$. So, if we make-believe about some predator and prey, we haven't thereby make-believed about sharks, rays, squid, cod, and lobster in the Adriatic Sea. Second, one might deny that modeling is a form of indirect representation. For example, one might claim that models are "statements with adjustable parameters" which when properly specified, then the model can be true of some target of interest. Models just are abstract or idealized representations

prey and predator randomly associate in accordance with the "law of mass action" they approximate "perfect mixing." Thus, the spatial arrangement is given by the model description. 
and presumably are a species of familiar genus. Of course, we can study those statements independently of any application if we like but they insofar as they are representational they represent targets in the world. Consider an analogy. Pablo Picasso's Les Demoiselles d'Avignon can be viewed and appreciated independently of whether this depiction abstracts or idealizes five prostitutes. But, we might be interested in that art historical question too.

Finally, Weisberg correctly notes unlike Maynard Smith, modelers do not always begin with, 'Imagine that...' Often they will simply stipulate that certain expressions stand for objects and properties as Karlin and Feldman do when they write, "Let's $x_{1}, x_{2}, x_{3}$ and $x_{4}$ her the frequencies of chromosomes $A B, A b, a B$, and $a b$ respectively, and $r$ the recombination fraction" (Karlin and Feldman, 1969, 65). When adding cover for prey in a predator prey model, Maynard Smith avoids the 'imagine that' locution,

Suppose that some number $x_{r}$ of the prey can find some cover or refuge which make them inaccessible to the predator. Volterra's equations without damping then become, $\dot{x}=a x-c y\left(x-x_{r}\right), \dot{y}=$ $-e y+c^{\prime} y\left(x-x_{r}\right)$. (Maynard Smith, 1989, 25)

But, not every game of make-believe explicitly begins 'Imagine that...', 'Pretend that...', or 'Make-believe that...' When we watch films, read novels, play games, etc. only rarely do we begin with such pronouncements. Likewise, we can begin a game of make-believe by using other locutions like, 'Let...' and 'Suppose...'. ${ }^{12}$ Consider children pretending they are superheroes. One might say, "Okay, you be Batman and I will be Superman." This is much like what Karlin and Feldman do above. So, I do not think modeling practice undercuts fictionalism about models.

I want to make one last point regarding fictionalism. Often Weisberg assumes that when one engages in make-believe or fiction, this requires that one forms a mental image of some state of affairs. If so, then one would have to explain how a mental representation is similar to some object or other (Cummins, 1989, Ch. 2). However, according to the fictionalists above, fiction and make-believe involve attitudes to propositions. We suppose that, make-believe that, imagine that, etc. If this is right, then we need not form

\footnotetext{
${ }^{12}$ I don't think however that whenever someone says, "Suppose..." we are engaging in make-believe. Likewise, I don't think saying that models contain assumptions suggests make-believe either any more than reductio ad absurdums or conditional proofs involve games of make-believe.
} 
any mental images regarding what the propositions are about. We might do this, but it is not required. And, if it is not required then it is not an objection against fictionalism.

\section{A Deflationary Approach to Models and Modeling}

Recent philosophical work on models and modeling arose out of analysis of analogy in science (Hesse, 1966; Campbell, 2013), and a critique of theories as axiomatic systems (Suppe, 1977; Suppes, 1960; van Fraassen, 1980). On the one hand, scientists use analogs to understand and predict features of systems. Waves of water are like sound waves, particles are like billiard balls, etc. As Mary Hesse suggested, scientists look for positive, negative, and neutral analogies between one thing and another. On the other hand, many philosophers thought that theories could not be axiomatic systems according to the Received View of Theories. For example, if axiomatic systems are relative to a language, then an axiomatized evolutionary theory in English would be distinct from an axiomatized evolutionary theory in French. But, axiomatizing then misses the real structure of scientific theories. The thought was, what really matters is not the language in which the theory is expressed but the objects or structures that make it true. Thus, the Semantic View of Theories claimed scientific theories are metalogical models. More liberal model-based views appeared too. For example, some claim that scientific theories are models which satisfy model descriptions and hypotheses which are of the form, " $M$ is similar to $S$ in a certain respects and to certain degrees." However, this has very awkward implications. If theories are pairs $<$ model, hypotheses $>$, then theories are not truth-apt; they are neither true nor false. And, if theories are neither true nor false, then they cannot believed, confirmed, make-believed to be true, etc (Chakravartty, 2001). My worry with recent literature on models and modeling is that models have been reified and the "philosophically irrelevant technical questions" that worried Bas van Fraassen are answered with "solutions to purely self-generated problems" (van Fraassen, 1980, 56). Weisberg's work keeps contact with scientific practice and its concerns and is rarely subject to this worry, but I do worry that this is true of some philosophical writings on model and modeling.

Let me sketch an alternative deflationary approach (Callender and Co- 
hen, 2009; Downes, 1992), which utilizes some of the resources Weisberg has provided which I very much admire. Moreover, like Weisberg, I think scientist's intentions are extremely important; my account like Weisberg's is a "Generalized Griceanism" (Callender and Cohen, 2009). ${ }^{13}$ Scientists use various vehicles to represent the world including concrete objects, equations, graphs, pictures, etc. These representational vehicles and the content they express are the models. Weisberg writes,

While the Lotka-Volterra model itself is not a set of equations, it can be studied only through proxies such as these equations. This is probably the main reason that scientists often informally refer to equations as models; their attention is focused on these equations. (Weisberg, 2013, 136-7)

Here I disagree; I think when a student is asked in a mathematical modeling class to "write down a model" that is what they are doing. We devise those representations by abstracting or idealizing target systems, which as Weisberg argues are abstractions from phenomena (c.f. Bogen and Woodward (1988)). When representing, we abstract when properties of the target are not included in the representation and idealize when we introduce in our representation properties that the target does not have. Galilean, minimalist, and multiple model idealizations all begin here. Moroever, scientists use those vehicles to further various ends such as understanding (P-GENERALITY, 1CAUSAL, SIMPLICITY), anticipating (MAXOUT), and intervening (1-CAUSAL) in the natural world. These representational vehicles can be more or less accurate; some of them are truth-apt. As a simple example, suppose we want to represent density-dependent population growth of a population of organisms in general. We say, "Let ' $N$ ' stand for the population abundance, let ' $r$ ' stand for its rate of increase independent of other species, and ' $K$ ' represent the maximum number of organisms an environment in general permits." We can then use mathematical formalisms, in this instance, calculus, to represent how this population in general changes with respect to time,

$$
\frac{d N}{d t}=r N\left(1-\frac{N}{K}\right)
$$

\footnotetext{
${ }^{13}$ Generalized Griceanism is committed to the idea that utterance or sentence meaning can be understood in terms of speaking meaning and this can be understood naturalistically in other terms.
} 
This equation represents populations. It does not represent a mathematical object which represents populations. And that is as it should be since if for every mathematical model description denotes a mathematical model, then inconsistent mathematical model descriptions would have to describe impossible objects.

Now, we can study this representation and it properties independently of whether or not it is true or false of populations and their growth rates. Modeling can be indirect insofar as we analyze and investigate models independently of testing or evaluating them for their truth. However, we can also determine if they are true, or as I prefer to say, "true enough" (Elgin, Teller). A model is true enough when it is imprecisely true with regard to appropriate fidelity criteria. For example, suppose we estimate our parameters $r$ and $K$ giving us $r^{*}$ and $K^{*}$ and place confidence intervals on them $r^{*} \pm x_{1}$ and $K^{*} \pm x_{2}$. As a result, then (4) is transformed into,

$$
\left(r^{*} \pm x_{1}\right) N\left(1-\frac{N}{\left(K^{*} \pm x_{2}\right)}\right)
$$

Our abstract model can be imprecisely true of populations. For various reasons (Odenbaugh, 2005), we may choose to not work with true enough models. False models can further a variety of aims by providing simple baselines, conceptual frameworks, etc (Odenbaugh, 2005). Additionally, we may simply be interested in finding the best fitting model through some measure of goodness-of-fit or model selection criterion. We need not articulate a measure of similarity de novo because statisticians have already developed them. This is no problem for scientific realism provided that when we aim to truthfully represent the system of interest we can show there are true enough models available that could do the work (Wimsatt, 1987). No scientific realist should think that representational accuracy is the only aim of science. Additionally, we can devise families of models varying parameter values, functional forms, or representational frameworks and see if there are robust theorems that follow from independent models. That is, we can see if the families exhibit parameter, structural, or representational robustness. If we can replace idealizations in models of family with true enough assumptions, we can show that those idealized models are harmless (Odenbaugh, 2011). What about those idealizations as opposed to abstractions? These are properties the model represents the system as having which it does not. Our models cannot be imprecisely true with respect to them; if a model im- 
plies it has target has property when it doesn't then it is false. However, as Wimsatt (1987) has noted, our representations are "piecewise approximations to reality"; Giere (1988) notes models are similar in certain respects and to certain degrees. But that is because parts of models can be imprecisely true even when they are false as a whole. We need not be interested in our model being imprecisely true or more generally representationally accurate in its entirety.

This the briefest summary of a deflationary approach which recognizes that models and modeling are ubiquitous. Modelers exploit ordinary practices that are found not only in the sciences but in the arts and ordinary life. We need not reify models and modeling; they are extensions of representation and communication tools we use all the time. And this sketch is in part an extension of Weisberg's excellent and thought-provoking Simulation and Similarity.

\section{References}

Benacerraf, P. (1965). What numbers could not be. The Philosophical Review 74 (1), 47-73.

Bogen, J. and J. Woodward (1988). Saving the phenomena. The Philosophical Review $97(3)$, 303-352.

Callender, C. and J. Cohen (2009). There is no special problem about scientific representation. Theoria. Revista de Teoría, Historia y Fundamentos de la Ciencia 21(1), 67-85.

Campbell, N. R. (2013). Physics: the elements. Cambridge University Press.

Chakravartty, A. (2001). The semantic or model-theoretic view of theories and scientific realism. Synthese 127(3), 325-345.

Cooper, J. M. and D. Hutchinson (1997). Complete works. Hackett Publishing.

Cummins, R. (1989). Meaning and mental representation.

Downes, S. M. (1992). The importance of models in theorizing: A deflationary semantic view. In PSA: Proceedings of the biennial meeting of the philosophy of science association, pp. 142-153. JSTOR. 
Field, H. H. (1980). Science without numbers: A defence of nominalism. Princeton University Press Princeton.

Frigg, R. (2010). Models and fiction. Synthese 172(2), 251-268.

Giere, R. N. (1988). Explaining science: A cognitive approach. University of Chicago Press.

Hellman, G. (1989). Mathematics without Numbers: Towards a ModalStructural Interpretation: Towards a Modal-Structural Interpretation. Oxford University Press.

Hesse, M. B. (1966). Models and analogies in science, Volume 7. University of Notre Dame Press Notre Dame.

Hughes, R. (1997). Models and representation. Philosophy of Science, S325S336.

Karlin, S. and M. W. Feldman (1969). Linkage and selection: new equilibrium properties of the two-locus symmetric viability model. Proceedings of the National Academy of Sciences 62(1), 70-74.

Levy, A. (2012). Fictional models de novo and de re.

Lewis, D. (1978). Truth in fiction. American Philosophical Quarterly 15(1), $37-46$.

Maynard Smith, J. (1989). Evolutionary genetics. Oxford University Press.

Odenbaugh, J. (2005). Idealized, inaccurate but successful: A pragmatic approach to evaluating models in theoretical ecology. Biology and Philosophy 20(2-3), 231-255.

Odenbaugh, J. (2011). True lies: Realism, robustness, and models. Philosophy of Science 78(5), 1177-1188.

Resnik, M. D. (1997). Mathematics as a Science of Patterns. Oxford University Press.

Shapiro, S. (1997). Philosophy of mathematics: Structure and ontology. Oxford University Press. 
Suppe, F. (1977). The structure of scientific theories. University of Illinois Press.

Suppes, P. (1960). A comparison of the meaning and uses of models in mathematics and the empirical sciences. Synthese 12(2), 287-301.

Thomson-Jones, M. (2010). Missing systems and the face value practice. Synthese 172(2), 283-299.

Toon, A. (2012). Models as make-believe: Imagination, fiction and scientific representation. Palgrave Macmillan.

van Fraassen, B. C. (1980). The scientific image. Oxford University Press.

Walton, K. (1990). Mimesis as make-believe: On the foundations of the representational arts. Harvard University Press.

Weisberg, M. (2013). Simulation and similarity: using models to understand the world. OUP USA.

Wimsatt, W. C. (1987). False models as means to truer theories. Neutral models in biology, 23-55. 